\title{
Effects of Processing on Electric Response of Carbon Black Filled Poly(methyl methacrylate) Composites against Organic Solvent Vapors
}

\author{
Xian Ming Dong, Ruo Wen FU, Ming Qiu ZHANG,,${ }^{\dagger}$ Zhi Pei QIN, \\ Bing ZHANG, and Min Zhi RONG* \\ Key Laboratory for Polymeric Composite and Functional Materials of Ministry of Education, \\ Zhongshan University, Guangzhou 510275, P. R. China \\ *Materials Science Institute, Zhongshan University, Guangzhou 510275, P. R. China
}

(Received August 12, 2003; Accepted September 29, 2003)

\begin{abstract}
Carbon black filled poly(methyl methacrylate) (CB/PMMA) composites were fabricated by solution mixing and polymerization filling, respectively. The effects of processing conditions on electrical conductivity of the composites and their electric responsivity against organic solvent vapors were investigated. The experimental results showed that molecular weight of the polymer matrix, carbon black content, and the composite film thickness greatly influence the response behaviors of the composites in solvent vapors. Furthermore, the composites prepared by polymerization filling have higher gas sensitivity, response rate, recovery rate, and reproducibility as compared to the composites by solution mixing. The sensing performance of the composites is found to be closely related to the microstructure of the materials, which provides possibilities for further improve the overall properties of the composites by altering the processing parameters.

KEY WORDS Carbon Black / Poly(methyl Methacrylate) / Conductive Polymer Composites /

Gas Sensor / Processing /
\end{abstract}

Dispersion of carbon black into an insulating polymeric matrix yields a conductive composite material characterized by a sharp decrease of its electrical resistivity when the filler content is increased above a critical value called percolation threshold. ${ }^{1,2}$ One of the most attractive features of these composites lies in their positive temperature coefficient (PTC) effect, ${ }^{3,4}$ which describes a switching from low to high resistance as temperature approaches the melting point of the matrix, and can be used to produce polymer based selfregulating heaters, microswitches, etc. In fact, a similar change has also been observed in the composites when facing organic solvents or vapors..$^{5-8}$ Absorption of the solvent or vapor into the composite is believed to cause a significant variation in electrical resistance by influencing the length of percolation paths between carbon black particles within the composite due to swelling or dissolution of the polymer matrix. ${ }^{9,10}$ On the basis of this feature, the composites are capable of acting as chemiresistors or electronic noses to detect, distinguish, and quantify various solvents or solvent vapors and might find applications in chemical, medical, automotive, food, and fragrances. ${ }^{11-13}$ So far, it is known that the sensing materials have many advantages, including easy fabrication with cost effectiveness, stability in many different environments, rapid response rate and high sensitivity to the targets, high selectivity, and miniature design.
With respect to the study on the conductive composites as chemisensors in the aspect of materials development, however, not many published reports are available because it is a relatively new area. Tsubokawa and co-workers ${ }^{6,14-16}$ modified carbon black surface by grafting polymerization, and found that the content and dispersity of the fillers, and crystallinity and molecular weight of the matrix notably influence the electrical responsivity, reproducibility and stability of the composites against organic vapors. Narkis et al. ${ }^{8,17}$ indicated that the sensitivity of carbon black filled immiscible polymer blends to organic solvents depends on blending composition, nature of the constituting components, interfacial feature, and production shear level. In our previous works, ${ }^{18-20}$ polymerization filling was employed to synthesize amorphous polymers based composites, which exhibit sufficient vapor sensibility and reproducibility. The content of carbon black, testing temperature and organic solvent species exert important influences on the electrical response of the composites.

To collect more information of the conductive composites for practical applications and to improve the performance of the composites, the present paper investigates the effects of processing parameters on the electric response behavior of carbon black/poly(methyl methacrylate) (CB/PMMA) composites by changing molecular weight of the matrix, filler concentration, 
composites thickness, and technique of composites manufacturing. PMMA is selected because of its amorphous characteristics. It is hoped that much more amorphous polymers can serve as matrices of conductive composites having sensibility to organic vapors, so that the potential candidate spectrum for chemisensors would be greatly broadened.

\section{EXPERIMENTAL}

\section{Materials}

Conductive carbon black (XC-72) supplied by Cabot Inc., with a surface area of about $254 \mathrm{~m}^{2} \mathrm{~g}^{-1}$ and a diameter of $50-70 \mathrm{~nm}$, was dried in a vacuum at $110^{\circ} \mathrm{C}$ for $48 \mathrm{~h}$ before use. The monomer, methyl methacrylate (MMA), was distilled for two times and the initiator, benzoyl peroxide (BPO), was purified by recrystallization from chloroform and methanol prior to the polymerization.

\section{Preparation of CB/PMMA Composites}

Two approaches were applied to the preparation of CB/PMMA composites, i.e., solution mixing and polymerization filling. In the case of solution mixing, homemade PMMA by radical polymerization with number average molecular weight, $M_{\mathrm{n}}$, of $7.42 \times 10^{4}$ or $1.94 \times 10^{4}$ was dissolved in $\mathrm{CHCl}_{3}$ at a polymer/solvent ratio of 1:6 (w/w). Then, CB was added into the solution at the desired proportion. Having been treated by ultrasonic agitation for $1 \mathrm{~h}$, the mixture was stirred with a magnetic stirrer at room temperature for 3-4 $\mathrm{h}$ to produce paste composites.

With respect to polymerization filling, the steps proceeded as follows. Typically, $2.0 \mathrm{~g}$ of $\mathrm{CB}, 18.0 \mathrm{~g}$ of MMA and a small amount of BPO were added into a $100 \mathrm{~mL}$ flask with a reflux condenser. Having been treated by ultrasonic agitation for $1 \mathrm{~h}$, the mixture was stirred at $90-95^{\circ} \mathrm{C}$ under nitrogen for $8 \mathrm{~h}$. After having been dried in a vacuum at room temperature for $48 \mathrm{~h}$, the product was weighed and the monomer conversion rate was calculated. Afterwards, the product was added into $20 \mathrm{~mL} \mathrm{CCl}_{4}$ and stirred for $1-2 \mathrm{~h}$ at room temperature to produce pasty composites. Then the quantitative product, which had been dried in a vacuum at room temperature for $48 \mathrm{~h}$, was dispersed in tetrahydrofuran (THF) and centrifuged at $1.6 \times 10^{4} \mathrm{rpm}$ for $30 \mathrm{~min}$. The supernatant solution was removed by decantation. The procedures were repeated until no more polymer could be detected in the supernatant layer. The molecular weight of the polymer was analyzed by gel permeation chromatography (GPC).

\section{Performance Measurement}

Specimen preparation and the corresponding measurements of electrical resistance and electrical responses to saturated organic solvent vapors were described in our previous works in detail. ${ }^{18}$ The responsivity is described by the ratio of the transient resistance to the initial resistance in air.

To observe the dispersion status of the conductive filler in the matrix polymer, a JSM-6330F scanning electronic microscope (SEM) was employed. The samples were obtained by freeze-fracture, and then gold sputtered prior to the examination.

\section{RESULTS AND DISCUSSION}

\section{Solution-blended CB/PMMA Composites}

It is known that the mechanical and physical properties of a conducting thermoplastic composite are mostly dominated by the nature of the filler material, whereas polymer matrix determines to a great extent the environmental characteristics of the composite. Since the CB/PMMA composites act as sensing materials in organic solvent vapors in the present work, the influence of matrix property should be discussed at the beginning. Figure 1 shows the composites' electrical resistivity as a function of CB content. Clearly, the resistivity of the composites with higher molecular weight matrix is about 1-3 orders of magnitude higher than that with lower molecular weight matrix at the same CB concentration. It means that the electric conduction paths can be established more easily in the low molecular weight PMMA. Because the composites were prepared via solution mixing, the flowability of the matrix polymer or the mobility of the macromolecular chains of the matrix in the solvent determines to a great extent the effect of compounding. The infiltration of the low molecular weight polymer solution into porous $\mathrm{CB}$ agglomerates would be more efficient than that of the high molecular

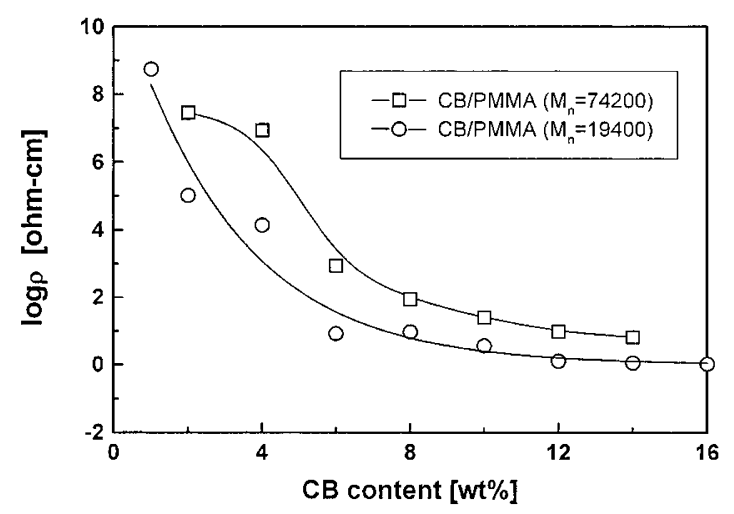

Figure 1. Electrical resistivity, $\rho$, of solution-mixed $\mathrm{CB} /$ PMMA composites as a function of $\mathrm{CB}$ content. The number average molecular weights of the matrix, $M_{\mathrm{n}}$, are given in the legends. 


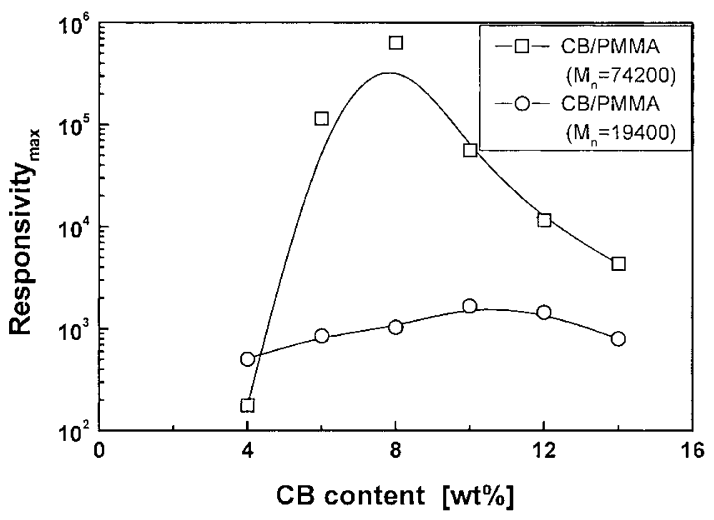

Figure 2. Dependence of maximum electrical responsivity of solution-mixed CB/PMMA composites on $\mathrm{CB}$ content in ethyl acetate vapor at $25^{\circ} \mathrm{C}$. The number average molecular weights of the matrix, $M_{\mathrm{n}}$, are given in the legends.

weight polymer solution. Therefore, smaller amount of $\mathrm{CB}$ particles is enough to form conduction networks throughout the composites in this case.

To further understand the effect of matrix molecular weight, dependence of the maximum electrical responsivity of the composites against ethyl acetate vapor is given in Figure 2. It is seen that the composites with higher molecular weight matrix are much more sensitive to the vapor than those with low molecular weight matrix over the entire $\mathrm{CB}$ content range of interests. The highest responsivity appears at about $8 \mathrm{wt} \%$ of $\mathrm{CB}$ in the former composites, approaching $6 \times 10^{5}$. The result is in agreement with that reported by Tsubokawa et al., ${ }^{16}$ who supposed that the formation of honeycomblike pores in the $\mathrm{CB} /$ polymer composites after absorbing a solvent vapor differs with different molecular weights of the matrices, and results in the different responses of the composites to the same solvent vapor. Here the authors believe that the polymer matrix with higher molecular weight possesses higher swelling capacity, which generates greater inter-particulate gaps of $\mathrm{CB}$ in the composites or breaks more conduction paths when the specimens are exposed to organic vapors. However, the molecular weight of the matrix cannot be increased unlimitedly for purposes of improving the electric responsivity of the composites. Excessively high molecular weight of the polymer will greatly increase intermolecular interaction and reduce its swelling power. In this context, lower molecular weight matrix would result in higher responsivity to organic vapors instead. ${ }^{19}$ Therefore, there would be an optimum molecular weight specified for each polymer serving as the matrix of the chemisensor. In other words, the matrix polymer of conducting composites used as gas sensors should possess appropriate molecular weight to ensure sufficiently high response and response rate.

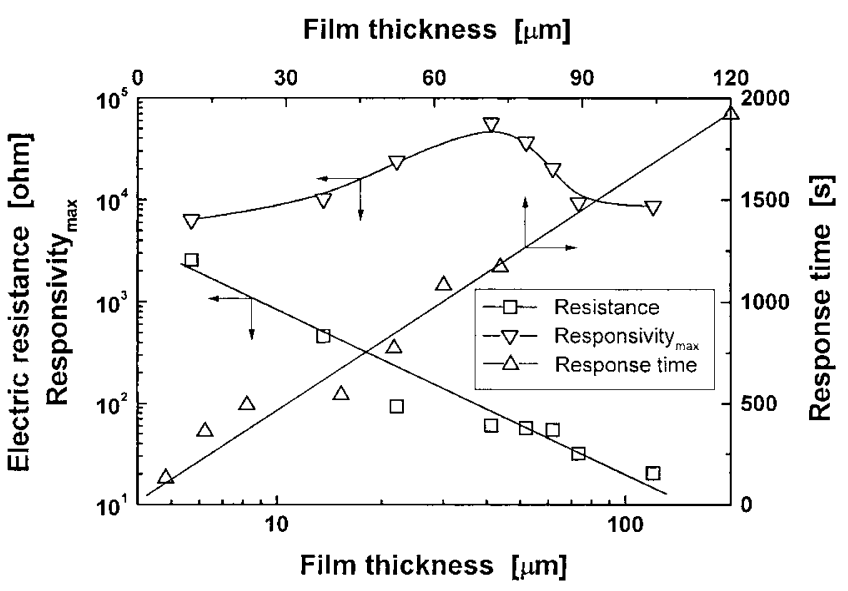

Figure 3. Effect of composite film thickness on initial resistance, maximum electrical responsivity and response time of solution-mixed CB/PMMA composites. $M_{\mathrm{n}}$ of PMMA: $7.42 \times 10^{4}$; $\mathrm{CB}$ content: $10 \mathrm{wt} \%$; Solvent vapor: ethyl acetate; Testing temperature: $25^{\circ} \mathrm{C}$.

From the viewpoint of practical application, thickness of the composite film is an important parameter related to the sensor performance because the thinner the composite film, the faster the response to organic vapor. ${ }^{14}$ As illustrated in Figure 3, there is a linear relationship between the response time (defined as the time needed to reach the maximum responsivity) and film thickness. It not only proves the conventional consideration of the response behavior of the composites, but also implies that Case II diffusion of the solvent molecules in the composites plays the leading role, as characterized by the constant response rate. For Fickian diffusion, the equilibrium absorption time is proportional to the square of the total film thickness. Evidently this is not the case here. That means relaxation processes occur in the polymer matrix for the polymer chains to respond to an osmotic swelling pressure and to rearrange themselves to accommodate the penetrant molecules. It again evidences that the mechanism responsible for the gas sensitivity of the composites is governed by the matrix swelling or volume expansion induced reversible conduction paths damage.

In addition, Figure 3 also shows the dependences of initial resistance and maximum responsivity on composite film thickness. The variation of composites initial resistance with the film thickness follows ohmic law, demonstrating the microstructural homogeneity of all the composites studied in the present work. It is interesting to note that the maximum responsivity of the composites against ethyl acetate vapor does not keep constant with a rise in composite film thickness, but peaks up at certain thickness. Similar phenomena were also observed in $\mathrm{CB} /$ poly(ethylene oxide) (PEO) composites in chloroform vapor. ${ }^{14}$ Usually a thicker composite film corresponds to more conduction pathways 
Table I. Effect of polymerization conditions on the gas sensitivity of CB/PMMA composites prepared by in-situ polymerization of methyl methacrylate in the presence of $\mathrm{CB}^{\mathrm{a}}$

\begin{tabular}{lccccccc}
\hline Sample ID & 1 & 2 & 3 & 4 & 5 & 6 & 7 \\
\hline Temperature $\left({ }^{\circ} \mathrm{C}\right)$ & $80-85$ & $90-95$ & $100-105$ & $90-95$ & $90-95$ & $90-95$ & $90-95$ \\
Time (h) & 6 & 6 & 6 & 4 & 6 & 8 & 6 \\
BPO $(\mathrm{wt} \%)$ & 2 & 2 & 2 & 2 & 1 & 2 & 3 \\
Monomer conversion (\%) & 61.0 & 68.0 & 76.0 & 36.7 & 10.7 & 86.8 & 84.6 \\
CB content $(\mathrm{wt} \%)^{\mathrm{b}}$ & 14.9 & 13.6 & 12.4 & 22.2 & 46.2 & 11.1 & 11.2 \\
$M_{\mathrm{n}}\left(\times 10^{4}\right)$ & 5.96 & 6.18 & 4.00 & 6.42 & - & 7.43 & 3.72 \\
Polydispersity index & 2.34 & 2.41 & 1.26 & 2.38 & - & 2.22 & 1.34 \\
Responsive time $(\mathrm{s})$ & 2340 & 1500 & 2280 & 1440 & 120 & 2190 & 1110 \\
Responsivity $_{\max }\left(\times 10^{4}\right)$ & 1.39 & 2.53 & 9.46 & 1.75 & $1.6 \times 10^{-4}$ & 8.40 & 22.2 \\
\hline
\end{tabular}

${ }^{\mathrm{a}}$ Dosages of $\mathrm{CB}$ and MMA are $1.0 \mathrm{~g}$ and $9.0 \mathrm{~g}$, respectively; Solvent vapor: $\mathrm{CH}_{3} \mathrm{COOC}_{2} \mathrm{H}_{5}$; Testing temperature: $30{ }^{\circ} \mathrm{C}$. ${ }^{\mathrm{b}}$ Calculated according to the monomer conversion.

inside, and hence greater probability of partial discontinuance of the contact $\mathrm{CB}$ when being put into an organic vapor. The maximum electric responsivity should increase with increasing the film thickness. However, the thicker composites might also present impedance to the diffusion of solvent vapor because the absorbed fluid is subjected to large internal pressure. The latter factor eventually reduces the amount of the absorbed solvent and the broken conduction networks as well. Consequently, the maximum responsivity is lowered in the case of thick composites. Competition between the two opposite factors yields the peak-like dependence of maximum responsivity of the composites on the thickness.

\section{CB/PMMA Composites Synthesized by Polymerization Filling}

The above analysis reveals that composites' electrical responsiveness is greatly influenced by the matrix characteristics and composites structure. Intimate contacts between the conductive filler particulates and the matrix would help to improve the gas sensitivity of the composites as matrix swelling directly controls the magnitude of increase in composites resistance. According to this consideration, polymerization filling is applied to produce CB/PMMA composites through insitu polymerization in the presence of $\mathrm{CB}$ particles. ${ }^{18}$ To have deeper understanding of the performance of the composites manufactured in this way, effects of various reaction conditions should be examined. From Table I it can be found that the monomer conversion increases with a rise in reaction time, temperature and initiator content. This coincides with the regular law observed in free radical polymerization. Since the present paper is not focused on the polymerization chemistry of the composites synthesis, a brief remark can be drawn instead. That is, the polymerization conditions seem not to greatly influence the sensitivity of the resultant composites. Most CB/PMMA composites exhibit high

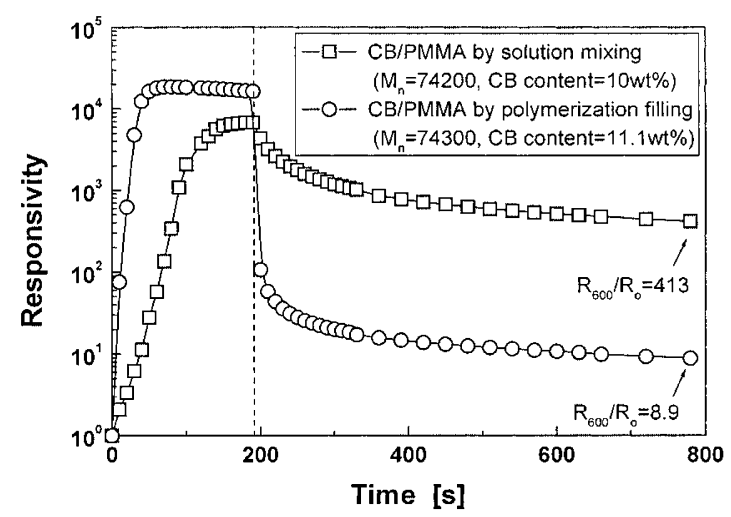

Figure 4. Electrical responses of CB/PMMA composites prepared by the two methods against chloroform vapor at $30^{\circ} \mathrm{C}$. The dash line defines the vapor absorption and desorption zones. $\mathrm{R}_{600}$ denotes electrical resistance of the composites after being moved out of the vapor for $600 \mathrm{~s}$ and $\mathrm{R}_{\mathrm{o}}$ the initial resistance, respectively. The ratio $R_{600} / R_{0}$ characterizes recovery rate of the composites.

responsivity ranging $10^{4}-10^{5}$ in ethyl acetate vapor. It means that the composites are provided with wide processing window as viewed from their application as gas sensing materials.

The response behaviors of CB/PMMA composites with similar $\mathrm{CB}$ content prepared by polymerization filling and solution mixing are compared in Figure 4. Besides the higher responsivity of the former one, the most obvious differences lie in the rates of response and recovery. Herein the resistance recovery rate of the composites is defined as $R_{600} / R_{0}$, where $R_{600}$ denotes electrical resistance of the composites after being moved out of the vapor for $600 \mathrm{~s}$ and $\mathrm{R}_{\mathrm{o}}$ the initial resistance, respectively. Figure 4 indicates that the material synthesized by polymerization filling has shorter response time and quicker resistance recovery rate than that by solution mixing. For example, the values of response time and $\mathrm{R}_{600} / \mathrm{R}_{0}$ of the former composites are $80 \mathrm{~s}$ and 8.9 , while those of the latter are $180 \mathrm{~s}$ and 413 , respectively. These differences must be originated from the different microstructures of the composites. 


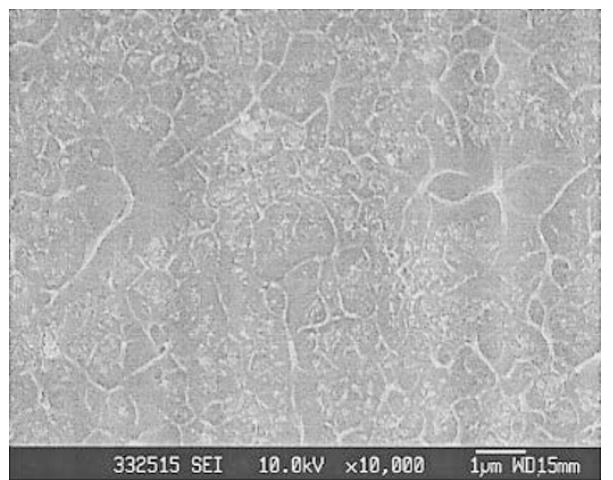

(a)

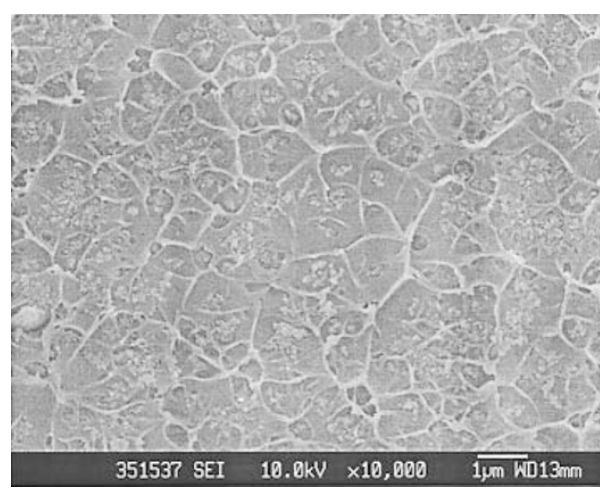

(b)

Figure 5. SEM micrographs of CB/PMMA composites prepared by (a) solution mixing $\left(M_{\mathrm{n}}\right.$ of PMMA: $7.42 \times 10^{4}$; CB content: $10 \mathrm{wt} \%)$, and (b) by polymerization filling $\left(M_{\mathrm{n}}\right.$ of PMMA: $7.43 \times 10^{4}$; CB content: $\left.11.1 \mathrm{wt} \%\right)$.

In the case of polymerization filling, the low molecular weight monomers are able to penetrate into the agglomerated $\mathrm{CB}$ prior to the reaction. As a result, relatively even distribution of smaller $\mathrm{CB}$ aggregates in the matrix and closer filler/matrix contacts can be obtained in the subsequent polymerization process. In contrast, it is somewhat difficult to breakdown the agglomerated CB in the course of solution mixing due to the limited shear force and high molecular weight nature of the readymade polymer. Large CB agglomerates have to appear in the resultant composites. As shown by Figure 5 (a), there are some regions in which completely no CB particles can be found in the composites by solution mixing. Comparatively, similar segregation is not perceivable in the composites by polymerization filling (Figure 5 (b)). It means that the amount and/or size of resinrich and filler-rich phases in the polymerization filling made composites are smaller than those in the solutionmixed composites. When being exposed to saturated solvent vapors, the absorption induced matrix swelling and $\mathrm{CB}$ particles disconnection would be faster in the former composites. For the same reason, the solvent desorption and reconstruction of the conduction paths are also faster in the former ones.

Performance reproducibility is another important

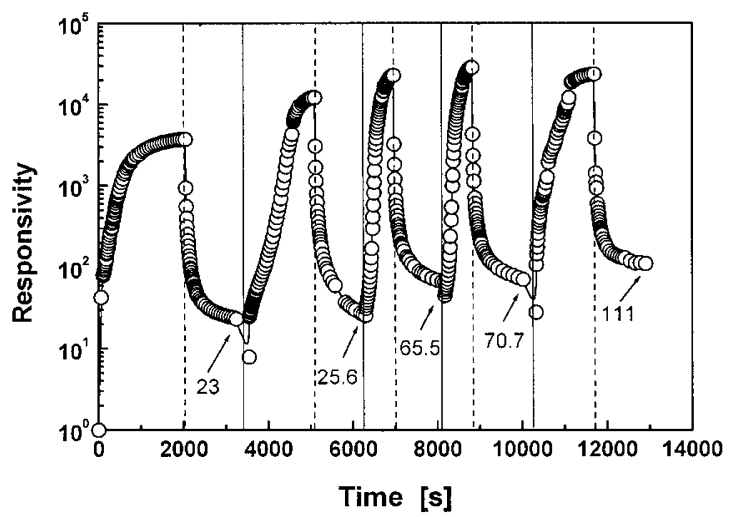

Figure 6. Electrical response of CB/PMMA composites prepared by solution mixing against tetrahydrofuran vapor at $30^{\circ} \mathrm{C}$ ( $M_{\mathrm{n}}$ of PMMA: $7.42 \times 10^{4}$; CB content: $10 \mathrm{wt} \%$ ). The dash lines define the vapor absorption and desorption zones.

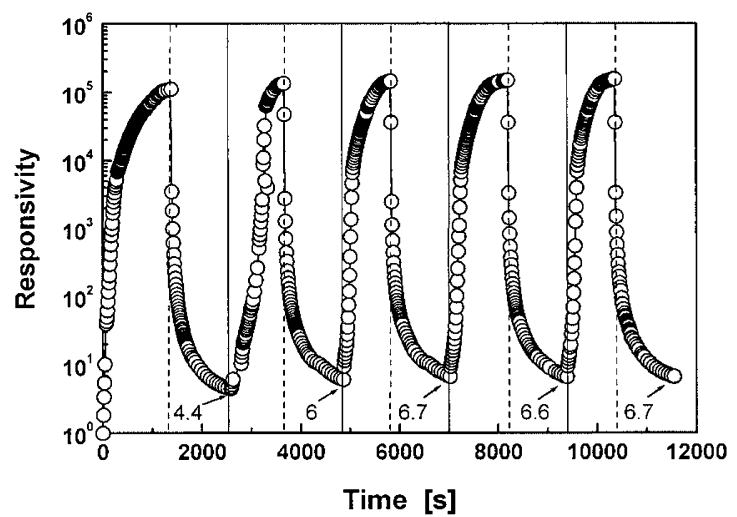

Figure 7. Electrical response of CB/PMMA composites prepared by polymerization filling against tetrahydrofuran vapor at $30^{\circ} \mathrm{C}\left(M_{\mathrm{n}}\right.$ of PMMA: $7.43 \times 10^{4}$; CB content: $\left.11.1 \mathrm{wt} \%\right)$. The dash lines define the vapor absorption and desorption zones.

property of the composites serving as gas sensors. Figures 6 and 7 further compare the electrical responses of the composites prepared by the two methods against tetrahydrofuran vapor. It is seen that the response of the composites prepared by solution mixing is unstable within the five absorption-desorption cycles. The maximum responsivity of the composites gradually increases with the repeated swelling-deswelling processes. Moreover, the resistance recovery rate is quite slow. Having been moved out of the vapor for $1200 \mathrm{~s}$ for each test cycle, the electrical resistance of the composites over the initial value $\left(\mathrm{R}_{1200} / \mathrm{R}_{\mathrm{o}}\right)$ increases from 23 to 111 within the five runs (Figure 6). On the contrary, after the first run of the absorption-desorption test, the electrical responses of the composites prepared by polymerization filling against tetrahydrofuran vapor have reached a stable status, exhibiting good reproducibility (Figure 7). The maximum responsivity of the composites is over $10^{5}$ for each test. In addition, the recovery rate is quite fast and almost constant that ensures similar ratios of $R_{1200} / R_{0}$ for each run of the 
test. It is therefore believed that solution mixing has brought about certain inherent structural defects in the composites, which are not healed during the repeated swelling-deswelling tests but aggravated slightly. For the case of polymerization filling, structural integrity of the composites is greatly improved and hence reproducible gas sensibility is observed.

\section{CONCLUSIONS}

Based on the above study, the following statements can be made.

1. Molecular weight of matrix polymer greatly influences electrical response of CB/PMMA composites in organic vapors. Depending upon swelling capacity of the polymers, the composites with matrices possessing different molecular weights exhibit different maximum responsivities.

2. The vapor transportation mode in CB/PMMA composites belongs to Case II diffusion. Due to the accumulated internal pressure in thick specimens that obstructs further vapor absorption, the increasing tendency of the maximum responsivity with increasing composites film thickness is changed as characterized by a peak-like dependence. It actually provides a criterion for the future device making in terms of sensor size.

3. In comparison with the composites prepared by solution mixing, the ones by polymerization filling have higher responsivity, response rate, recovery rate and reproducibility when exposed to organic vapors. Therefore, the latter approach is more suitable for manufacturing gas sensors in practice.

4. CB/PMMA composites prepared in this work are able to respond to various solvent vapors. The selectivity of their response performance can be acquired by either using the approach of vapor detector arrays ${ }^{5}$ or quantifying the vapor concentration dependence of resistance. ${ }^{21}$

Acknowledgments. The financial support by the National Natural Science Foundation of China (Grant 50133020), the Team Project of the Natural Science Foundation of Guangdong (Grant 20003038), and the
Talent Training Program Foundation of the Higher Education Department of Guangdong Province are gratefully acknowledged.

\section{REFERENCES}

1. M. Q. Zhang and H. M Zeng, In: Handbook of Thermoplastics, O. Olabisi Ed., Marcel Dekker Inc., New York, 1997, p. 873-891.

2. F. Lux, J. Mater. Sci., 28, 285 (1993).

3. G. Yu, Zhang, M. Q. Zhang, and H. M Zeng, J. Appl. Polym. Sci., 70, 559 (1998).

4. M. Q. Zhang, G. Yu, H. M. Zeng, H. B. Zhang, and Y. H. Hou, Macromolecules, 31, 6724 (1998).

5. B. J. Doleman, M. C. Lonergan, E. J. Severin, T. P. Vaid, and N. S. Lewis, Anal. Chem., 70, 4177 (1998).

6. J. Chen and N. Tsubokawa, Polym. Adv. Technol., 11, 101 (2000).

7. F. Zee and J. W. Judy, Sensor Actuat. B-Chem., 72, 120 (2001).

8. M. Narkis, S. Srivastava, R. Tchoudakov, and O. Breuer, Synth. Met., 113, 29 (2000).

9. A. Marquez, J. Uribe, and R. Cruz, J. Appl. Polym. Sci., 66, 2221 (1997).

10. N. Tsubokawa, Y. Shirai, M. Okazaki, and K. Maruyama, Polym. Bull., 42, 425 (1999).

11. M. Okazaki, K. Maruyama, M. Tsuchida, and N. Tsubokawa, Polym. J., 31, 672 (1999).

12. J. Chen, H. Iwata, N. Tsubokawa, Y. Maekawa, and M. Yoshida, Polymer 43, 2201 (2002).

13. K. J. Albert, N. S. Lewis, C. L. Schauer, G. A. Sotzing, S. F. Stitzel, T. P. Vaid, and D. R. Walt, Chem. Rev, 100, 2595 (2000).

14. J. Chen and N. Tsubokawa, Polym. J., 32, 729 (2000).

15. J. Chen and N. Tsubokawa, J. Macromol. Sci., Pure Appl. Chem., A38, 383 (2001).

16. J. Chen, N. Tsubokawa, Y. Maekawa, and M. Yoshida, Carbon, 40, 1602 (2002).

17. S. Srivastava, R. Tchoudakov, and M. Narkis, Polym. Eng. Sci., 40, 1522 (2000).

18. X. M. Dong, R. W. Fu, M. Q. Zhang, B. Zhang, J. R. Li, and M. Z. Rong, Carbon, 41, 371 (2003).

19. J. R. Li, J. R. Xu, M. Q. Zhang, and M. Z. Rong, Macromol. Mater. Eng., 288, 103 (2003).

20. X. M. Dong, R. W. Fu, M. Q. Zhang, B. Zhang, J. R. Li, and M. Z. Rong, Polym. Bull., 50, 99 (2003).

21. X. M. Dong, R. W. Fu, M. Q. Zhang, B. Zhang, and M. Z. Rong, J. Polym. Sci., Polym. Phys. Ed. (submitted) 